\title{
Retrotransposons and siRNA have a role in the evolution of desiccation tolerance leading to resurrection of the plant Craterostigma plantagineum
}

\author{
Tobias Hilbricht ${ }^{1}$, Serena Varotto ${ }^{2}$, Vittorio Sgaramella ${ }^{3}$, Dorothea Bartels ${ }^{4}$, Francesco Salamini ${ }^{3}$ and \\ Antonella Furini ${ }^{5}$ \\ ${ }^{1}$ Max-Planck-Institut fur Züchtungsforschung, Carl-von-Linne Weg 10, D-50829 Köln, Germany; ${ }^{2}$ Dipartimento di Agronomia Ambientale e Produzione \\ Vegetale, University of Padova, Agripolis-Viale dell’Università 16, 35020 Legnaro, Padova, Italy; ${ }^{3}$ Fondazione Parco Tecnologico Padano, CERSA, via Einstein \\ - Località Cascina Codazza, 26900 Lodi, Italy; ${ }^{4}$ Molekulare Physiologie und Biotecnologie der Pflanzen, University of Bonn, Kirschallee 1, D-53115 Bonn, \\ Germany; ${ }^{5}$ Dipartimento Scientifico e Tecnologico, University of Verona, Strada Le Grazie 15, 37134 Verona, Italy
}

\section{Summary}

Author for correspondence: Antonella Furini

Tel. +390458027950

Fax +390458027929

Email: antonella.furini@univr.it

Received: 15 February 2008

Accepted: 10 March 2008
- Craterostigma plantagineum can lose up to $96 \%$ of its water content but fully recover within hours after rehydration. The callus tissue of the plant becomes desiccation tolerant upon pre-incubation with abscisic acid (ABA). In callus and vegetative organs, $A B A$ addition and water depletion induce a set of dehydrationresponsive genes.

- Previously, activation tagging led to the isolation of Craterostigma desiccation tolerant (CDT-1), a dehydration-related $A B A$-inducible gene which renders callus desiccation tolerant without $A B A$ pre-treatment. This gene belongs to a family of retroelements, members of which are inducible by dehydration.

- Craterostigma plantagineum transformation with mutated versions of CDT-1 indicated that protein is not required for the induction of callus desiccation tolerance. Northern analysis and protoplast transfection indicated that CDT-1 directs the synthesis of a double-stranded 21-bp short interfering RNA (siRNA), which opens the metabolic pathway for desiccation tolerance.

- Via transposition, these retroelements have progressively increased the capacity of the species to synthesize siRNA and thus recover after dehydration. This may be a case of evolution towards the acquisition of a new trait, stimulated by the environment acting directly on intra-genomic DNA replication.

Key words: abscisic acid (ABA), Craterostigma plantagineum, desiccation tolerance, retrotransposon, siRNA.

New Phytologist (2008) 179: 877-887

(c) The Authors (2008). Journal compilation @ New Phytologist (2008)

doi: 10.1111/j.1469-8137.2008.02480.x

\section{Introduction}

The resurrection plant Craterostigma plantagineum can tolerate up to $96 \%$ loss of its relative water content and recover from such extreme dehydration within several hours (Bartels \& Salamini, 2001 and Supplementary Material Fig. S1). By contrast, callus tissue of this plant has a strict requirement for exogenous abscisic acid (ABA) in order to survive severe water loss (Bartels et al., 1990 and Supplementary Material Fig. S1), and water depletion and $\mathrm{ABA}$ treatment result in the induction of the same set of dehydration- and ABA-responsive genes (Bartels et al., 1990). T-DNA activation tagging has allowed the isolation of callus lines tolerant to desiccation even in the absence of ABA (Furini et al., 1997; Smith-Espinoza et al., 2005). The first gene isolated from these lines, Craterostigma desiccation tolerant (CDT-1; Furini et al., 1997), when transcribed at a high level in the callus as a result of the nearby insertion of the Agrobacterium tumefaciens gene 5 
promoter ( $\mathrm{pg} 5)$, induces ABA- and dehydration-responsive genes represented in this plant by the genes encoding the cDNA pc6-19, pc11-24 and pc27-45 (Bartels et al., 1990).

$C D T-1$ is an unusual gene: (1) the longest open reading frame (ORF) starting with ATG is followed by 20 triplets and ends with TAA; (2) no translation products have been observed; (3) no sequences comparable to that of CDT-1 have been found in databases; (4) a poly(A) tract of 17-22 nucleotides has been reported in the $5^{\prime}$ region of all cDNA and genomic clones, and a $3^{\prime}$ poly $(\mathrm{A})$ tail is present in genomic clones; (5) the structure of CDT-1 is reminiscent of that of retrotransposons; it is intronless, flanked by direct repeats and present in multiple copies in the genome; (6) most importantly, CDT-1 transcript has never been detected in fresh wild-type leaves; in the callus it is up-regulated by $A B A$, and in wild-type vegetative organs it is induced by dehydration and repressed by rehydration (Furini et al., 1997).

Retrotransposons are genetic elements that transpose through reverse transcription of RNA intermediates; in plants they play a significant role in genome structure, organization and evolution (Kumar \& Bennetzen, 1999; Brosius, 2003). The process of retrotransposition is highly invasive: retrotransposons do not excise from the genome and their copy number increases exponentially with transposition (Beguiristain et al., 2001). Plant genomes have mechanisms to control retrotransposition which limit the dangerous effects of new insertions, as well as excessive growth of the retrotransposon population (Liu \& Wendel, 2000). Plant retrotransposons quiescent during normal plant development may respond to environmental stress, tissue culture conditions and pathogen attack (Kalendar et al., 2000; Beguiristain et al., 2001). Active retrotransposons can affect transcription of adjacent genes: for wheat insertion sequence Wis 2-1A, a wheat Long-TerminalRepeats (LTRs)-retroelement, a direct link exists between retrotransposon transcription, readout activity from the LTRs and silencing or activation of the adjacent genes by means of antisense or sense RNA, respectively (Kashkush et al., 2003).

Transcriptional interference is also a mechanism by which retrotransposons might affect gene expression (Whitelaw \& Martin, 2001). In plants, the notion that short interfering RNAs (siRNAs) can be essential molecules for retrotransposon regulatory mechanisms is not new. The presence of siRNAs was investigated in three different plant retrotransposons (Hamilton et al., 2002): the tobacco (Nicotiana tabacum) LTR-retrotransposon transposon Nicotiana tabacum (Tnt1) and two short interspersed elements (SINEs), TS SINE of tobacco and AtSN1 of Arabidopsis. Small RNAs of both sense and antisense polarities were detected in equal abundance. Based on the size of these siRNAs, the authors suggest that retroelement siRNAs are produced by mechanisms similar, but not identical, to those involved in transgene RNA silencing. Because retrotransposons are often transcriptionally silent, it has been suggested that siRNAs play a role in transcription inactivation (Hamilton et al., 2002). Akin to DNA transcription into active siRNA (Mallory \& Vaucheret, 2006), SINE-like DNA sequences could be first transcribed into single-stranded RNA (ssRNA) and then converted into functional double-stranded siRNA. In plants, this would lead to the formation of transacting (ta) siRNA (Dunoyer et al., 2005; Yoshikawa et al., 2005).

In this study we investigated the role of CDT-1 in the pathway for desiccation tolerance. First we provide evidence that $C D T-1$ translation is not required, and then we show that an siRNA is responsible for opening the ABA and desiccation tolerance pathways. We lastly suggest that the ability to survive extreme conditions may have been stimulated by the environment acting on intra-genomic duplication of DNA elements.

\section{Materials and Methods}

\section{Plant material, constructs and transformation}

Craterostigma plantagineum Hochst. plants were maintained and propagated in vitro as previously described (Bartels et al., 1990). The transformation procedure and the media for callus induction and maintenance were as reported by Furini et al. (1994), and identification of desiccation-tolerant calli, including untreated calli (negative control) and ABA-treated calli (positive control), was performed as detailed in Furini et al. (1997). ABA treatments were performed by adding $10 \mu \mathrm{M}$ ABA for $4 \mathrm{~d}$ to in vitro grown calli and plants.

The Agrobacterium tumefaciens strain GV3101 pMP90RK (Koncz \& Schell, 1986) was used as the host strain for the pPCV811 (Koncz et al., 1994). An EcoRI-HindIII fragment containing the A.tumefaciens gene 5 promoter (pg5) and the CDT-1 cDNA (accession number Y11822) was cloned adjacent to the hygromycin-resistance $(h p t)$ gene into the pPCV811 vector (control construct a) (Furini et al., 1997). In addition, three mutated forms of CDT-1 (constructs $\mathrm{b}, \mathrm{c}$ and d) were cloned under the control of pg5 into pPCV811 and used for C. plantagineum transformation (Fig. 1a). In construct b, the triplet ATG was changed to GGG, in construct $\mathrm{c}$ the $3^{\prime}$ end was deleted up to nucleotide 424 and in construct $\mathrm{d} 30 \mathrm{bp}$ were deleted between positions 131 and 160 . In constructs $a, b$ and $\mathrm{d}$ the native $C D T-1$ poly(A) tail is recognized as a termination signal. In construct $\mathrm{c}$ transcription termination relies on the nopaline synthase polyadenylation site present in the pPCV811 vector downstream of CDT-1 insertion (Koncz et al., 1994). All mutated forms of $C D T-1$ were generated using the mutagenesis kit from Amersham Biosciences (Buckinghamshire, UK), with the CDT-1 cDNA as template. Agrobacterium tumefaciens transformation was achieved by high-voltage electroporation (Wen-jun \& Forde, 1989). Transformation with the different constructs was confirmed by PCR analysis of DNA isolated from transformed calli. PCR primers were designed between the CDT-1 gene and $p g 5$. To exclude problems of plasmid contamination, a PCR reaction was performed with primers designed outside of the T-DNA. Primers and conditions were as described previously (Furini 
New

Phytologist

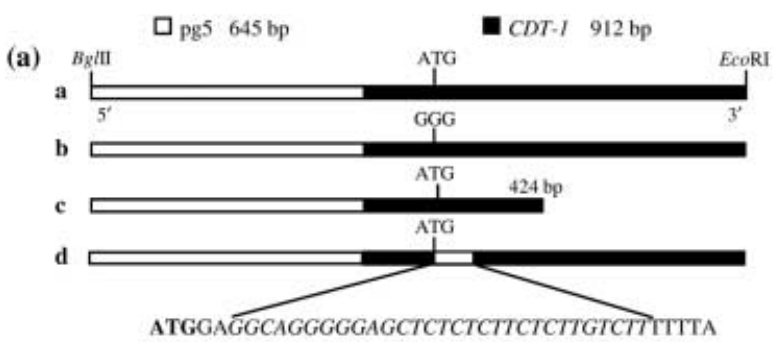

(b)

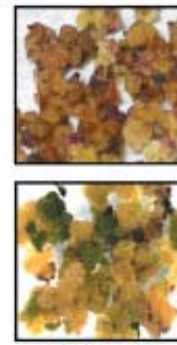

Hydrated
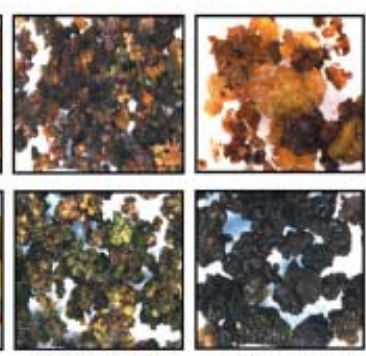

Dehydrated

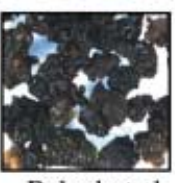

Rehydrated

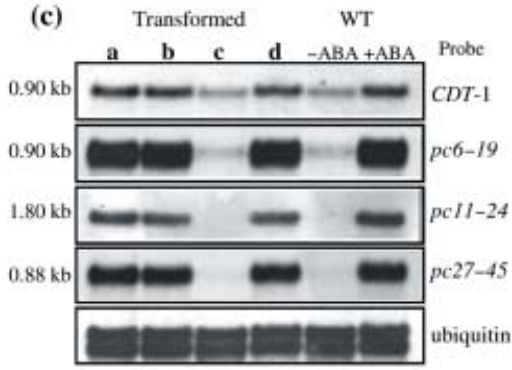

Fig. 1 Craterostigma desiccation tolerant (CDT-1) gene expression activates the desiccation pathway in Craterostigma plantagineum. (a) Constructs used for transformation. Construct a: full-length CDT-1 cDNA (Furini et al., 1997). Construct b: the ATG was mutated into GGG. Construct c: a 424-bp CDT-1 missing 488 bp at the 3'-end. Construct d: a CDT-1 cDNA with a 30-bp deletion. (b) Desiccation-tolerant calli (upper panels) transformed with construct $b$ displayed a reddish color typical of abscisic acid (ABA)-treated wild-type calli. Similar phenotypes were observed in calli transformed with constructs a and d. Desiccation-sensitive calli (lower panels) transformed with construct c had a yellowgreenish color typical of untreated wild-type calli (Supplementary Material Fig. S4). (c) RNA blot analysis of poly(A)+ RNA (2 $\mu g$ per lane) isolated from calli transformed with constructs $a, b, c$ and $d$, and from wild-type untreated and ABA-treated calli. The transcription level is reported for CDT-1 and for genes encoding cDNA pc6-19, pc11-24, and pc27-45.

et al., 1997). Calli resistant to hygromycin and tested for effective transformation were 13,13,10 and 9 for constructs a, b, c and d, respectively; fresh callus material was sampled from several independent callus lines.

\section{Nucleic acid extraction and analysis}

Poly(A) ${ }^{+}$RNA extraction and RNA gel blot analysis were as described by Bartels et al. (1990). Total RNA was extracted from calli using Trizol reagent (Invitrogen, Carlsbad, CA, USA). Genomic DNA was isolated using the Qiagen DNeasy Plant Mini Kit (Qiagen, Hilden, Germany). Probes for RNA blot were CDT-1 cDNA and the $C$. plantagineum cDNA clones from genes $p c 6-19, p c 27-45$ and $p c 11-24$ (Bartels et al., 1990). To test for the presence of sense and antisense CDT-1 transcripts, $30 \mu \mathrm{g}$ of total RNA extracted from leaves and callus were subjected to RNA blot hybridization analysis. Probes were CDT-1 cDNA labeled by specific priming incorporation of $\left[\alpha-{ }^{32} \mathrm{P}\right] \mathrm{dCTP}$. For strand-specific probes, $100 \mathrm{ng}$ of CDT- 1 cDNA was combined with $1 \mu \mathrm{g}$ of RT-CDT-1-A primer (5'-AAGATACAGGGCTAAATAGCC-3') or RT-CDT-1-T primer (5'-TTGTGCCGCCACCACCACCGT-3') for sense or antisense DNA labeling, respectively, in a total volume of $14 \mu \mathrm{l}$ and DNA was denatured at $95^{\circ} \mathrm{C}$ for $5 \mathrm{~min} ; 2.5 \mu \mathrm{l}$ of $0.5 \mathrm{mM}$ dNTP mix (minus dCTP), $2.5 \mu$ of Klenow buffer, $1 \mu \mathrm{l}(5 \mathrm{U})$ of Klenow DNA polymerase and $5 \mu \mathrm{l}$ of $\left[\alpha_{-}{ }^{32} \mathrm{P}\right] \mathrm{dCTP}$ $(50 \mu \mathrm{Ci})$ were then added to the mixture to a final volume of
$20 \mu$ l. Incubations were for $1 \mathrm{~h}$ at $37^{\circ} \mathrm{C}$. Probes were purified with MicroSpin ${ }^{\mathrm{TM}} \mathrm{G} 50$ columns from Amersham Bioscience.

\section{In situ hybridization}

In situ hybridization experiments were carried out as described in Varotto et al. (2003). In situ hybridization was performed on wild-type ABA-treated calli, calli transformed with construct a, and roots and leaves of ABA-treated plants. The tissues were fixed in $4 \%$ paraformaldehyde and $0.2 \%$ glutaraldehyde in $0.1 \mathrm{M}$ phosphate buffer $(\mathrm{pH} 7.2)$ for $16 \mathrm{~h}$ at $4^{\circ} \mathrm{C}$ and embedded in Paraplast Plus (Sigma-Aldrich, St Louis, MO, USA). Sections $(7-10 \mu \mathrm{m})$ were cut using the microtome RM 2135 (Leica, Nussloch, Germany) and collected on xylane-coated slides. Slides were deparaffinized, treated with $5 \mu \mathrm{g} \mathrm{ml}^{-1}$ proteinase $\mathrm{K}$ and hybridized with sense and antisense riboprobes in $50 \%$ formamide at $50^{\circ} \mathrm{C}$ overnight. CDT-1 cDNA was cloned into the pGem-T vector (Promega, Madison, WI, USA) and transcribed in vitro to obtain DIGUTP (Roche Applied Science, Mannheim, Germany) labeled RNA probes using T7 and Sp6 RNA polymerases. As control, DIG-labeled sense and antisense riboprobes were transcribed using a 450-bp cDNA fragment of the pc27-45 gene cloned into the pGem-T vector (Promega). After hybridization the slides were extensively washed in $2 \times$ saline sodium citrate (SSC) at $50^{\circ} \mathrm{C}$ and treated with $20 \mu \mathrm{g} \mathrm{ml}^{-1}$ RNaseA (Roche Applied Science). DIG detection and signal visualization 
were carried out using NitroBlue Tetrazolium (NBT) and 5bromo-4-chloro-3-indolyl phosphate (BCIP) staining following the manufacturer's instructions (Roche Applied Science). Images were acquired using the DC 300F camera (Leica).

\section{Small RNA analysis}

Small RNA analysis was carried out as described by Hamilton \& Baulcombe (1999), with slight modifications. Total RNA was extracted from wild-type ABA-treated and -untreated calli, and calli transformed with construct a, using Trizol reagent (Invitrogen). The RNA pellet was resuspended in $0.5 \mathrm{ml}$ of sterile diethyl pyrocarbonate (DEPC)-treated water, heated at $65^{\circ} \mathrm{C}$ for $5 \mathrm{~min}$ and placed on ice. Polyethylene glycol 8000 (5\%)- $0.5 \mathrm{M} \mathrm{NaCl}$ was added, and the RNA preparation was kept on ice for $30 \mathrm{~min}$ and then pelleted by centrifugation at $10000 \mathrm{~g}$ for $10 \mathrm{~min}$. The supernatant enriched with low-molecular-weight RNA was passed through Qiagen purification columns (Qiagen) and the low-molecularweight RNA was precipitated by adding a $1 / 10$ volume of $3 \mathrm{M}$ sodium acetate, $\mathrm{pH} 5.2$, and 3 volumes of absolute ethanol. After incubation for $2 \mathrm{~h}$ at $-20^{\circ} \mathrm{C}$, the precipitate was centrifuged, washed with $70 \%(\mathrm{v} / \mathrm{v})$ ethanol and dissolved in an appropriate volume of sterile DEPC-treated water. Small RNAs were separated on a $15 \%$ polyacrylamide $-7 \mathrm{M}$ urea gel, transferred onto Hybond $\mathrm{N}^{+}$membrane by electroblotting for $1 \mathrm{~h}$ at $100 \mathrm{~V}$ in $0.5 \times$ Tris/Borate/EDTA (TBE), and then fixed by cross-linking with UV light. Ten pmol of 20-nt oligoribonucleotide 5'-UGUGCCGCCACCACCACCGU-3' and 5'-AAGAUACAGGGCUAAAUAGC-3', designed based on the regions from 215 to $234 \mathrm{bp}$ and from 845 to 864 bp of the CDT-1 cDNA sequence, respectively (Supplementary Material Fig. S3), was included in the same gel as a size and polarity control.

${ }^{32} \mathrm{P}$-labeled riboprobes were obtained by in vitro transcription with either T3 or T7 polymerase (Promega) and $\left(\alpha-{ }^{32} \mathrm{P}\right)$-CTP $(50 \mu \mathrm{Ci}$ per $20 \mu \mathrm{l}$ reaction volume; specific activity $400 \mathrm{Ci} \mathrm{mmol}^{-1}$ ), using the linearized plasmid containing full-length CDT-1 cDNA as a template to produce sense or antisense RNA probes. The labeled RNA was hydrolyzed by the addition of an alkaline buffer $(80 \mathrm{mM}$ sodium bicarbonate and $120 \mathrm{mM}$ sodium carbonate) to $20 \mu \mathrm{l}$ of the reaction volume followed by incubation of the mix at $60^{\circ} \mathrm{C}$ for $2.5 \mathrm{~h}$. The incubation time was calculated as previously described (Mette et al., 2000). The solution was neutralized with $20 \mu \mathrm{l}$ of $3 \mathrm{M}$ sodium acetate, $\mathrm{pH}$ 5.2. Prehybridization and hybridization were performed as described by Hamilton \& Baulcombe (1999). The membrane was washed twice in $2 \times$ SSC and $0.2 \%$ sodium dodecyl sulphate (SDS) at $40^{\circ} \mathrm{C}$ for $30 \mathrm{~min}$ and exposed to Kodak film. In addition, the membrane was probed with several 21 mers: 5'-TAAGCAGCTCGCGTTGTCCGC-3' (from 435 to 455 bp), 5'-AGTTTGTGGGAGCTCGTCTCT-3' (from 532 to 552 bp), 5'-TGTGGAGCCCTCAGTCCAACG-3' (from 585 to $605 \mathrm{bp}$ ), 5'-TCAGTCCAACGTAGACAAAAC-3' (from 595 to $615 \mathrm{bp}$ ) and finally $5^{\prime}$-GTTAAAATGAAGAGTATCGTA-3" sense and 5'-TACGATACTCTTCATTTTAAC-3' antisense, designed based on the CDT-1 sequence. The latter sequence corresponds to the region from 634 to $654 \mathrm{bp}$ of the CDT-1 cDNA (Supplementary Material Fig. S3). The oligoprobes were labeled with $\left[\gamma^{32} \mathrm{P}\right] \mathrm{ATP}$ in the presence of T4 kinase.

\section{Protoplast isolation and electroporation}

Protoplasts were isolated from wild-type calli. Enzymatic digestion was performed in KM8p liquid medium (Kao \& Michayluk, 1975) supplemented with $2 \%$ cellulase 'Onozuka' R10, $1 \%$ macerozyme, $0.2 \mathrm{M}$ glucose and $0.2 \mathrm{M}$ mannitol, $\mathrm{pH} 5.5$, after incubation overnight at $25^{\circ} \mathrm{C}$ in the dark, with gentle shaking $(30 \mathrm{rpm})$. The average protoplast yield was $3 \times 10^{5}$ protoplasts per gram of callus. Protoplasts were sequentially filtered through two sieves $(300$ and $85 \mu \mathrm{m})$, washed in solution I $(0.4 \mathrm{M}$ mannitol and $3.6 \mathrm{mM} 2-(\mathrm{N}-$ morpholino)ethanesulfonic acid (Mes), $\mathrm{pH}$ 5.5) and resuspended in solution II (solution I plus $0.1 \mathrm{mM} \mathrm{CaCl}_{2}$ ) to a density of $2 \times 10^{6}$ protoplasts $\mathrm{ml}^{-1}$ and kept on ice for $1 \mathrm{~h}$ before electroporation. Protoplasts were then divided into four aliquots: (1) untreated control, (2) protoplasts for transfection with siRNA designed based on 595-615 bp of CDT-1 cDNA, (3) protoplasts for transfection with siRNA designed based on 634-654 bp of CDT-1 cDNA and (4) protoplasts treated with ABA $(10 \mu \mathrm{M})$ $36 \mathrm{~h}$ before harvesting. All protoplast samples underwent electroporation and were collected $36 \mathrm{~h}$ later. siRNAs corresponding to the regions 595-615 bp and 634-654 bp of the CDT-1 cDNA were designed with $5^{\prime}$ phosphate, $3^{\prime}$ hydroxyl, and two-base overhangs at the $3^{\prime}$ end of each strand as indicated previously (Krichevsky \& Kosik, 2002) and synthesized by Qiagen. The siRNA primer sequences corresponding to the region 634-654 bp were: sense strand $5^{\prime}$ UAAAAUGAAGAGUAUCGUAd(TT)- $3^{\prime}$ and antisense strand 5'-UACGAUACUCUUCAUUUUAd(AC)-3'. The siRNA primer sequences corresponding to the region 595$615 \mathrm{bp}$ were similarly prepared. Annealing for duplex siRNA formation was performed by incubation at $90^{\circ} \mathrm{C}$ for $1 \mathrm{~min}$ and at $37^{\circ} \mathrm{C}$ for $1 \mathrm{~h}$. Protoplasts (aliquots of $10^{6}$ ) were mixed with $3 \mu \mathrm{g}$ of siRNA in a cuvette with a $0.4-\mathrm{cm}$ gap and electroporated at $0.3 \mathrm{kV}$ and $125 \mu \mathrm{F}$ as described previously (Vanitharani et al., 2003). After electroporation, protoplasts were transferred to a new tube containing $1 \mathrm{ml}$ of solution II, kept on ice for $30 \mathrm{~min}$, collected by centrifugation at $70 \mathrm{~g}$ for $5 \mathrm{~min}$ and then cultured in $1 \mathrm{ml}$ of culture solution as described by Qi et al. (2004). The experiment was repeated three times.

\section{Quantification of gene-specific transcripts by real-time PCR}

Total RNA was isolated from protoplasts and collected $36 \mathrm{~h}$ post-electroporation using the RNeasy Plant Mini Kit 
(Qiagen). cDNA was prepared using ImProm-II ${ }^{\mathrm{TM}}$ Reverse Transcriptase as indicated by the manufacturer (Promega). Real-time PCR was performed with the ABI Prism sequence detection system (Applied Biosystems, Foster City, CA, USA) using the Platinum SYBR Green qPCR SuperMix UDG (Invitrogen). The following forward and reverse primers were designed based on cDNA sequences for genes $p c 27-$ 45, pc6-19 and pc11-24 (Bartels et al., 1990): pc27-45, forward 5'-TGAGCTTGATAAAGGACATCG-3' and reverse 5'-TGAGACAATGGAATCGTGAA-3'; $p c 6-19$, forward 5'-GGGATGAAGGACAAGATGAA-3' and reverse 5'-CCTTTTTCTCATGCTGCTGT-3'; pc11-24, forward 5'-AGTGATGGAGGGTCAAACTG-3' and reverse $5^{\prime}$ GGCTTGACCCATGTTTCTTA-3'. All data were normalized to a cDNA fragment of $t k t 3$, a transketolase gene constitutively expressed in C. plantagineum (Bernacchia et al., 1995), amplified in the same conditions using primers TKT3-forward (5'-GGCCACACTGATATTGCTTT-3') and TKT3-reverse (5'-TTAATAGCAGCGCGAATCTT- $\left.3^{\prime}\right)$. The data were organized according to the $2^{-\Delta \Delta C T}$ method for relative gene expression analysis (Livak \& Schmittgen, 2001).

\section{Isolation of genomic clones}

A genomic library was constructed from leaves of C. plantagineum as previously described (Furini et al., 1997). The library was amplified and $-5 \times 10^{5}$ plaques were screened using the CDT-1 cDNA as a probe.

\section{Results}

\section{CDT-1 translation is not required for desiccation} tolerance

Although CDT-1 translation products were not detected in previous work (Furini et al., 1997), the translation of the relevant ORF into a 21-amino acid peptide still remained a possibility. To test this hypothesis, $C$. plantagineum leaf discs were transformed with constructs carrying mutated versions of the wild-type CDT-1 cDNA fused to pg5 (Fig. 1a). Construct a was used as a control, as the overexpression of the wild-type CDT-1 cDNA in callus tissues led to the acquisition of desiccation tolerance and the expression of dehydration- and ABA-induced genes (Furini et al., 1997). Calli transformed with construct b were desiccation tolerant, acquired the typical color of ABA-treated calli (Fig. 1b, upper panels; compare with Supplementary Material Fig. S1) and expressed dehydration- and ABA-inducible genes (Fig. 1c). A similar phenotype was observed for calli transformed with construct $d$. In construct $b$, the hypothetical ATG starting codon was mutated into GGG. This change did not alter the desiccation tolerance of the transgenic callus. Construct $d$ contains a 30-bp deletion which drastically reduces the length of the hypothetical ORF and alters the sequence of the corresponding peptide, if any: still, it induced the transcription of dehydration-related genes and supported desiccation tolerance (Fig. 1c). Conversely, a version of CDT-1 truncated at position $424 \mathrm{bp}$, and thus missing $488 \mathrm{bp}$ of the $912 \mathrm{bp}$ of the CDT-1 cDNA, but still containing the ORF (construct c), neither elicited desiccation tolerance nor induced genes $p c 6-19, p c 11-24$ and $p c 27-45$ (Fig. 1c). Desiccation-sensitive calli showed a yellowish-green color (Fig. 1b, lower panels), like the wild-type ABA-untreated callus (phenotypes of calli transformed with constructs $a, b$, $\mathrm{c}$ and $\mathrm{d}$ are presented in Supplementary Material Fig. S4). Compared with c, constructs a, b and d gave a higher level of transcription of CDT-1; in calli transformed with construct c the level of $C D T-1$ transcription was similar to that of wild-type calli in the absence of ABA. This is probably a result of transcription in the callus of the native CDT-1 gene(s), as evident from Fig. 1c, lane c, where the size of the transcript corresponds to a $C D T-1 \mathrm{mRNA}$. In calli transformed with construct $\mathrm{c}$, other RNA signals were not detected by northern analysis. The absence of construct c-specific transcripts in northern analysis has been interpreted as a result of mRNA instability, possibly caused either by the lack of the poly(A) tail or by heterogeneous terminations. In conclusion, only the transcription level enhanced by pg 5 in transgenic calli, or by ABA in wild-type calli, is associated with the acquisition of desiccation tolerance, and this requires the presence of the 488-bp sequence (constructs a and b) or part of it (construct d), positioned at the $3^{\prime}$-end of the CDT$1 \mathrm{cDNA}$, but not the translation of the hypothetical ORF.

\section{Detection of CDT-1 sense and antisense RNA and in situ hybridization}

Transformation with $p g 5-C D T-1$ constructs allowed the detection of high levels of CDT-1 transcript in callus tissues, as pg5 is active only in tissue with a high level of internal auxin (Koerber et al., 1991). Furthermore, RNA blot analyses indicated that the transcribed region of CDT-1 was c. 900 bp (Furini et al., 1997 and Fig. 1c) and that signals of comparable sizes were detected for both strands (Fig. 2a). In differentiated leaves, CDT-1 transcript was detected throughout the dehydration process and suppressed as soon as water was supplied to dried leaves (Furini et al., 1997). In situ hybridization with CDT-1 RNA probes was carried out on callus and on differentiated plant tissues (Fig. 2b). In CDT-1overexpressing calli and in wild-type ABA-treated calli, the CDT-1 signal, with antisense probe, was localized specifically in cells with substantial cell wall thickening (Fig. 2b, panels 1, 2 and 3). CDT-1 signal on the same type of cells was also detected with sense probe in CDT-1-overexpressing calli (Supplementary Material Fig. S2, panel 3) and in wild-type ABA-treated calli (not shown). An attempt was made to localize CDT-1 transcript in a dehydrated plant. As desiccation is a process affecting both the nucleous and the cytoplasm, 
(a)

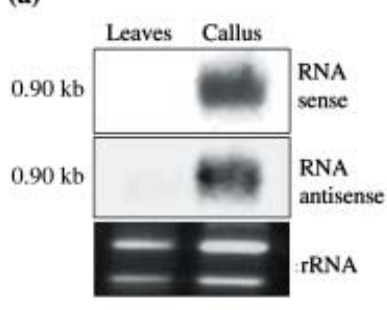

(b)

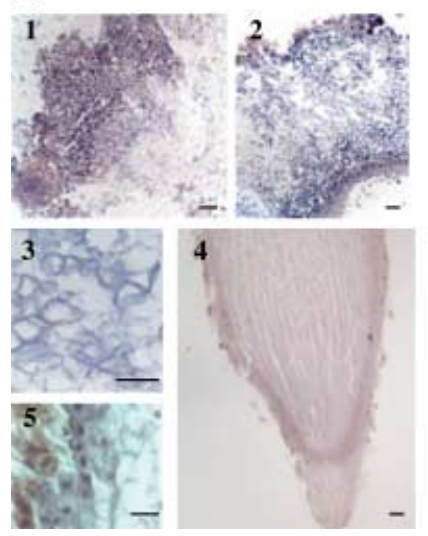

(c)

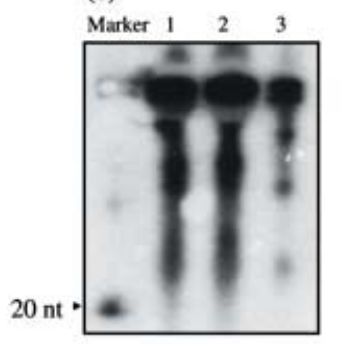

(e)

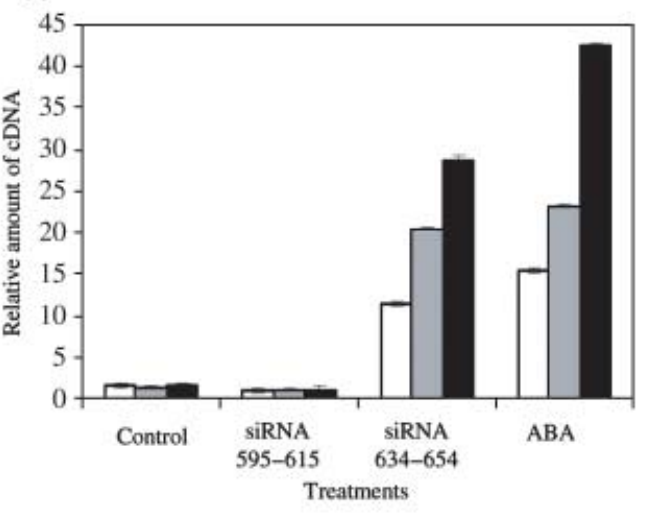

Fig. 2 Expression level, bistrandedness and transcription site of Craterostigma desiccation tolerant (CDT-1) and related desiccation-inducible marker genes. (a) RNA blot analysis of total RNA (30 $\mu \mathrm{g}$ per lane) extracted from Craterostigma plantagineum untreated leaves and from callus expressing CDT-1 and tested with sense and antisense CDT-1 RNA. (b) In situ hybridization using CDT-1 mRNA. Bars, $100 \mu \mathrm{m}$. (1, 2) CDT-1 antisense riboprobe on a section of (1) abscisic acid (ABA)-treated wild-type callus and (2) callus overexpressing CDT-1. (3) Higher magnification of (1) showing cells with an unusual cell wall; (4) longitudinal section of wild-type ABA-treated root hybridized with CDT-1 antisense riboprobe. (5) Higher magnification of (4). (c) RNA blot analysis of low-molecular-weight RNA hybridized with a hydrolyzed $C D T-1$ antisense probe. RNA was isolated from ABA-treated wild-type callus (lane 1), from callus transformed with construct a (lane 2) and from untreated wild-type callus (lane 3). The migration position of a 20-nt RNA is denoted by an arrow. (d) The filter in (c) was probed with sense and antisense 21 mers corresponding to positions 634-654 bp in the CDT-1 cDNA sequence. The migration position of a 20-nt RNA is indicated by an arrow. (e) Level of transcription of the desiccation- and ABA-induced genes pc27-45 (black bars), pc6-19 (gray bars) and pc11-24 (white bars), in callus-derived protoplasts measured by real-time PCR. Reported are the results obtained with untreated protoplasts (control), protoplasts transfected with short interfering RNA (siRNA) corresponding to 595-615 bp in the CDT-1 cDNA, protoplasts transfected with siRNA corresponding to 634-654 bp in the CDT-1 cDNA, and protoplasts incubated with $10 \mu \mathrm{M}$ ABA for $36 \mathrm{~h}$. Error bars denote SE.

the hybridization signal could not be detected in dried tissues; however, ABA-treated root apexes gave a strong signal restricted to a thin layer of parenchyma cells adjacent to the epidermis (Fig. 2b, panels 4 and 5). Experiments with wild-type ABA-untreated root apexes showed only minimal background signals (Supplementary Material Fig. S2, panels 4 and 5). To exclude the possibility that the detection of CDT-1 transcripts was a result of artefacts, ABA-treated wild-type callus was hybridized with a cDNA fragment of the $p c 27-45$ gene (Bartels et al., 1990). In this case only the antisense probe yielded positive signals (Supplementary Material Fig. S2, panels 1 and 2).

\section{Small RNA analysis}

The detection of sense and antisense CDT-1 RNA prompted us to test whether the desiccation-tolerant phenotype was mediated by small RNA. No specific size of low-molecular-weight RNA was detected with sense and antisense riboprobes obtained from in vitro transcription followed by hydrolysis of the CDT-1 template. Instead, a smear was observed (Fig. 2c), indicating the accumulation of RNA of variable length. After testing several oligonucleotides (see Materials and Methods) designed based on the 488-bp segment of CDT-1 deleted in construct c, positive results were obtained when low-molecularweight RNA was hybridized with sense and antisense 21 mers (GTTAAAATGAAGAGTATCGTA) corresponding to the region from 634 to $654 \mathrm{bp}$ in the CDT-1 cDNA (Supplementary Material Fig. S3). This oligonucleotide had some similarity to microRNA 159 (Achard et al., 2004), which is highly conserved in evolution. Both sense and antisense probes gave a hybridization signal with lowmolecular-weight RNA from desiccation-tolerant calli but no accumulation of small transcripts was evident when desiccation-sensitive wild-type calli were examined (Fig. 2d). We propose that this desiccation-dependent small RNA plays 
a regulatory role in the process leading to desiccation tolerance and should be considered an siRNA.

If this double-stranded RNA has an siRNA function, it should re-activate the genes induced by dehydration or ABA, when fed directly to wild-type callus-derived protoplasts. Craterostigma plantagineum wild-type callus-derived protoplasts were therefore transfected with the double-stranded CDT-1 siRNA corresponding to the GTTAAAATGAAGAGTATCGTA oligonucleotide sequence, from 634 to $654 \mathrm{bp}$ in the CDT-1 cDNA, which gave a strong signal in RNA blot analysis. The transient expression of dehydration-responsive genes, quantified by real-time PCR $36 \mathrm{~h}$ post-transfection, showed that protoplasts treated with siRNA (634-654 bp) expressed desiccation- and ABA-inducible genes, as did the ABA-treated protoplasts in the absence of siRNA. Conversely, desiccation- and ABA-responsive genes were not induced in control protoplasts nor in protoplasts transfected with siRNA derived from the region from 595-615 bp in the CDT-1 cDNA sequence (Fig. 2e). The induction of these genes in protoplasts transfected with a specific CDT-1 siRNA indicated that a mechanism of RNA interference may be responsible for the induction of the dehydration tolerance pathway in C. plantagineum.

\section{CDT-1 retrotransposition}

The demonstration that CDT-1 siRNA (634-654 bp) is expressed in desiccation-tolerant calli and is responsible for the induction of $\mathrm{ABA}$ - and dehydration-responsive genes prompted us to screen the genomic library to isolate CDT-1 homologs and to analyze the features of several genomic and cDNA clones. The structure of genomic clones (schematized in Fig. 3a) indicates that the CDT-1 elements (Fig. 3a, regions $\mathrm{b}$ and $\mathrm{c}$ ) are flanked by different DNA sequences characterized by direct repeats of 5 to $22 \mathrm{bp}$ (Fig. 3a,b, regions a and d). Furthermore, the length of the $3^{\prime}$ poly(A) tail (from 10 to $>60 \mathrm{bp}$; Fig. 3b, region c) and the presence of direct repeat core sequences (colored in Fig. 3b,c) appearing in more than one clone made it possible to reconstruct, at least in part, the temporal series of transpositions (Fig. 3c). Sequencing of CDT-1 cDNA clones revealed that several loci in $C$. plantagineum are transcribed into essentially identical CDT-1 mRNA. The derived cDNAs are almost invariant, except for the length of the $5^{\prime}$ oligo(A) sequence $(17,18,20$ or $21 \mathrm{bp}$ ), which was of 19,21 and $22 \mathrm{bp}$ in three different sequenced genomic clones.

\section{Discussion}

Retrotransposons are the most abundant class of transposable elements in plants (Feschotte et al., 2002). They are often considered as inert components of the genome, but their presence in databases of expressed sequence tags (Vicient et al., 2001; Nigumann et al., 2002) indicates that many have retained the capacity to initiate transcription. Moreover, the abundance of retrotransposons in most eukaryotic genomes (Lander et al., 2001; Feschotte et al., 2002), together with data indicating that retrotransposons are induced by various signals (Mhiri et al., 1997; Takeda et al., 1999), supports the idea of retrotransposons as controlling elements (Kashkush et al., 2003). It has also been reported that pseudogenes which do not produce functional full-length proteins are actively transcribed and regulate the expression of other related genes by mechanisms based on the functional significance of noncoding RNAs (Korneev et al., 1999; Hirotsune et al., 2003). The results of this study indicate that translation of the retroelement CDT-1 is not required for desiccation tolerance of the callus. However, the transcript of the $3^{\prime}$-end sequence of CDT-1 deleted in construct c (Fig. 1c) contains information necessary for desiccation tolerance. Another retroelement, $C D T-2$, isolated from a new T-DNA-generated desiccation-tolerant mutant, differs in sequence from CDT-1, but the highest sequence similarity between them is found in the region deleted in construct c. Furthermore, the CDT-2 sequence does not include the $5^{\prime}$ region of CDT-1 that may potentially code for a short peptide (Smith-Espinoza et al., 2005). In addition to being desiccation tolerant, transgenic calli overexpressing CDT-1 have a reddish color similar to wild-type ABA-treated calli, reinforcing the observation that a common response is elicited by CDT-1 overexpression and ABA. The obvious link between the two responses is that CDT-1 itself is ABA inducible (Furini et al., 1997). In fact, unlike vegetative organs of $C$. plantagineum, wild-type calli are not desiccation tolerant (Bartels et al., 1990). However, when they are treated with ABA, or transformed with $C D T-1$, they transcribe dehydration-related genes and become desiccation tolerant.

The analysis of the Arabidopsis genome indicates antisense RNA expression of $c$. 20\% of the pseudogenes annotated as not-expressed sequences (Yamada et al., 2003). Moreover, transposable elements have the potential to generate doublestranded RNA as a result of promoters in their terminal repeats and/or because of their random integration in the genome (Raizada et al., 2001). In our work, in situ hybridization revealed sense and antisense RNA signals, and RNA blot analysis confirmed that both strands of $C D T-1$ are transcribed. Low-molecular-weight RNA analysis and the activation of the desiccation and ABA pathway in protoplasts transfected with a double-stranded siRNA identified in the 488-bp region deleted in construct $c$ indicate that the siRNA resulting from CDT-1 transcription activates a desiccation-related response. We can speculate that the siRNA is processed from a longer double-stranded RNA precursor and represses gene expression through sequence-specific interactions with sequences of the C. plantagineum genome. The abundance of CDT-1 transcripts induced by ABA treatment, by dehydration in differentiated tissues and by the CDT-1 transgene in the callus may be recognized by the cell as a signal of stress and, 
(a)

CDT-1 element

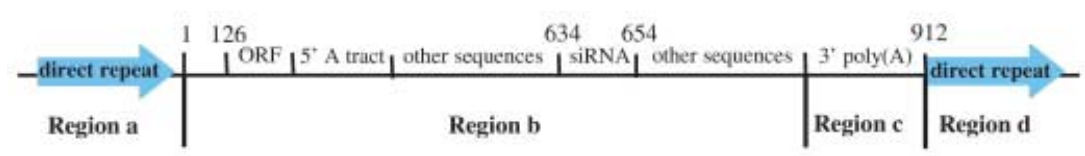

(b)

\begin{tabular}{|c|c|c|c|c|}
\hline Clone & Region a & Start of Region b & Region $c$ & Regiond \\
\hline 1: & TTCCATCGTTGAACAATGGCCTTCGAAAAAA & AAAAAACCCTT & $25 \mathrm{~A}$ & GAACAATGGCTTCGAAATT \\
\hline 2: & AAGAGIAGAGAGAATCTGATATACTCCACGCTIGTCTC & АAAAAACCCTT & $17 \mathrm{~A}$ & GAGAGAGAGAATCTGATGC \\
\hline 3: & 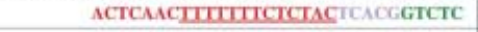 & AAAAAACCCTT & $15 \mathrm{~A}$ & TTITTTTCTCTATTCACA \\
\hline 4: & AАATGACACGTTTGAAATACCACTTGTCTC & AАAAAMCCCTT & $10 \mathrm{~A}$ & GAAATTACGCTTGTCTCGT \\
\hline 5: & ACTCAACTTITTTCTCTACTCACGGTCTC & AAAAAACCCTT & $15 \mathrm{~A}$ & СТTTTTTССТСТАСТСАCAAAG \\
\hline 6: & GGTATTGAATATTOTTGTATTTCTTCACGGTCTC & АААAAACCCTT & $10 \mathrm{~A}$ & GAATATTGTTGTATTTCTTTC \\
\hline 7: & TTCACTAGGAATGGACTCGAAATTACCOTTGTCTC & AAAAAACOCTT & $15 \mathrm{~A}$ & CTAGGIATTGGACTCAAGG \\
\hline 8: & GCAAGTATT & AAAAAACCCTT & 104 & GTATTAAGA \\
\hline 9: & TATCANGIAATAACCCCGAGAA & COCTCCCATCT & $>60 \mathrm{~A}$ & TAATAACCCCGAGAAGAA \\
\hline 10: & TGACACGTTTGAAATTACGCTIGTTCT & AAAAAACCCTT & $10 \mathrm{~A}$ & GAAATTACGCTTGTTCTCGTG \\
\hline
\end{tabular}

(c)
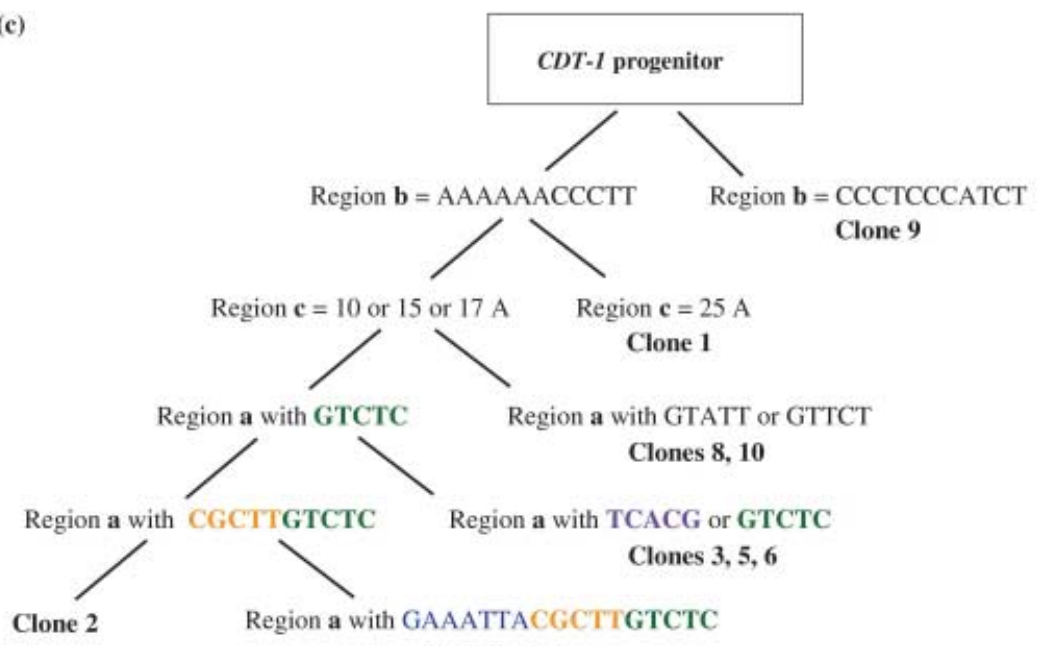

Clones 4,7

Fig. 3 Structure and transposition history of Craterostigma desiccation tolerant (CDT-1) elements. (a) Structure of the elements. The features are those of the CDT-1 clone previously described (Furini et al., 1997; Supplementary Material Fig. S3). Ten genomic clones had a similar structure, except for the modifications described in the text and in (b) of this figure. (b) DNA sequences of regions $a, b, c$ and d of 10 genomic clones harboring CDT-1 elements. Direct repeats flanking the CDT-1 element and present in regions a and d are underlined. Clone 9 was 25 bp shorter in region b. Clones 1 to 7 , with the exception of clone 4, had an expansion of variable size (3-18 bp) between the direct repeat in region $a$ and the start of region $b$. The expanded parts were not found in the sequenced cDNA clones except for the clone previously described (Furini et al., 1997; Supplementary Material Fig. S3). Colored bases in region a represent core motifs present in more than one clone. They made it possible to reconstruct, at least in part, the temporal series of transpositions shown (c). (c) Putative succession of transpositions starting from a common progenitor of $C D T-1$ present in 10 genomic clones. The elements start with region b (with $5^{\prime}$ expansions of 3 to 18 bp in clones $1,2,3,5,6$, and 7). Colored letters signify core motifs that are or have been parts of direct repeats. The sequence at the start of region $b$, the number of base pairs in the $3^{\prime}$ poly $(A)$ (region $c$ ), and the type of core motifs establish the succession of transposition events.

through the formation of double-stranded RNA, the transcripts may be converted to small RNAs which in turn might control the expression of genes responsible for desiccation tolerance to avoid deleterious stress effects on the cell. Because in $C$. plantagineum only a limited number of genes implicated in the revival of dried tissues have been identified, with respect to the complexity of the desiccation tolerance phenomenon, the gene(s) potentially recognized by CDT-1 siRNA is as yet unknown. The fact that CDT-1 siRNA was detected in transgenic callus as well as in ABA-treated wild-type callus suggests an identical role for $C D T-1$ in both types of tissue.
Many of the newly identified tiny RNAs are not expressed at distinct stages of development but only in particular cell types (Bartels, 2004). In the callus and in planta, CDT-1 expression is restricted to special cells or cell layers (in the case of the root); however, all cells from both the callus and the root are capable of resurrecting, making it likely that the effect of the CDT-1 gene or gene products can be transferred between cells or between organs. In plants, it is known that systemic signals with RNA components (Voinnet \& Baulcombe, 1997; Voinnet et al., 1998) convey RNA interference between cells. One component of this process is vascular dependent and transmits silencing signals between distinct organs. A 
second type of movement involves cell-to-cell contact via plasmodesmata and is based on reiterated short-distance signaling events involving 21-nt siRNA (Himber et al., 2003; Dunoyer et al., 2005). In specific cases, plant micro RNAs (miRNAs) or siRNAs are active when added exogenously to cultured plant cells (Vanitharani et al., 2003).

What is special in the case of CDT-1 siRNA is the functional link provided by the association of $C$. plantagineum retroelements with the increase in the level of their siRNA transcription. In establishing a mechanism capable of mediating between environment and DNA, the evolutionary relevance of the transposition of siRNA-carrying retroelements can hardly be assessed directly, but can be conjectured. The starting point is the consideration that CDT-1 mRNA accumulates in wild-type plants only during dehydration (Furini et al., 1997). This implies that the environment controls both the level of transcription of retroelements with potential siRNA activity and their genomic reinsertion. Plants do not have a sequestered germline: in meristematic cells new insertions of the element should contribute to increased desiccation tolerance in the progeny of the plant, provided that they can be transcribed under stress.

CDT-1 cDNAs vary only in the length of the $5^{\prime}$ oligo(A) sequence. Based on available data, a minimum of five CDT-1 elements are transcribed. This evidence was difficult to obtain because of problems with PCR amplification of genomic or transcribed CDT-1 elements across the region containing the $5^{\prime}$ A tract. Nevertheless, the four possible lengths of the $5^{\prime}$ oligo(A) sequence in CDT-1 cDNA (17, 18, 20 or $21 \mathrm{bp})$, together with the longest CDT-1 cDNA sequence already reported (Furini et al., 1997 and Supplementary Material Fig. S3), indicate that at least five CDT-1 genes are transcribed. However, a larger number is expected, based on the high frequency of CDT-1 elements in the genome, and the quasi invariance of their sequence as evident from the data on genomic clones. In sequenced cDNA clones the length of the $5^{\prime}$ poly(A) tract was $17,18,20$, or $21 \mathrm{bp}$; the same tract had a length of 19, 21 and $22 \mathrm{bp}$ in three different genomic clones. The multiplicity of CDT-1 transcripts is also supported by the mRNA analyses of dehydrated and rehydrated plants: a limited but clear size variability of CDT-1 transcripts has been observed (Furini et al., 1997). The sequence of genomic clones indicates that the $C D T-1$ genes are flanked by different DNA sequences. This, together with the high level of reiteration of the element in the genome, supports the conclusion that $C D T-1$ elements, after transcription under stress, are variably reintegrated in the $C$. plantagineum genome by retrotransposition. This is reinforced by the analysis of 10 such loci, which are all flanked by direct repeats of 5-22 bp. Furthermore, the presence of direct repeat core sequences and the different lengths of the $3^{\prime}$ poly(A) tails allowed, at least in part, reconstruction of the temporal series of transpositions that have increased the $C D T-1$ putative siRNA level. We underline the almost complete invariance of the CDT-1 sequence in the genomic clones so far considered. This is an unusual finding for plant transposons (Kumar \& Bennetzen, 1999) - as if selection acted to preserve the integrity of the siRNA motif and/or of the DNA information necessary for element retrotranscription, or for double-stranded RNA synthesis. Sequence invariance supports the acquisition of adaptive traits during evolution: this is a possibility suggested for miRNA-related gene control mechanisms (Voinnet, 2004). Moreover, for the CDT-1 family, acquisition is likely to have been rapid, given the absence in plant databases of transcribed CDT-1 relatives.

To conclude, the mechanism described here provides an evolutionary explanation of the interaction between environment and DNA: the higher the expression of the retrotransposon under dehydration, the more frequent its reinsertion into the genome. As a result, there is an increasing probability that the CDT-1 element will land in a DNA sequence capable of directing transcription under stress. When repeated, such a process should result, even without selection, in a progressively higher level of synthesis of the CDT-1 siRNA, which eventually triggers the onset of desiccation tolerance. In this respect, it is worth recalling the two components of Lamarck's model of organic change (Burkhardt, 1995): the 'power of life', or the natural progress of organic development, can be seen as the 'cause that tends to make organization increasingly complex'; and the modification of this progress by constraining circumstances suggests that 'they arise from the influence of the climate, variations in temperature of the atmosphere, ... from diversities of places, ... from habits of movements' (Lamarck, 1801, 1802).

\section{Acknowledgements}

The authors wish to dedicate this paper to their colleague and friend Dr Lorena Borgato in recognition of her invaluable contribution to the research prior to her untimely death earlier last year.

\section{References}

Achard P, Herr A, Baulcombe DC, Harberd NP. 2004. Modulation of floral development by a gibberellin-regulated microRNA. Development 13: 3357-3365.

Agarwala R, Aravind L, Bailey JA, Bateman A, Batzoglou S, Birney E, Bork P, Brown DG, Burge CB, Cerutti L et al. 2001. (International Human Genome Sequencing Consortium) Initial sequencing and analysis of the human genome. Nature 409: 860-912.

Bartel DP. 2004. MicroRNAs: genomic, biogenesis, mechanism, and function. Cell 116: 281-297.

Bartels D, Salamini F. 2001. Desiccation tolerance in the resurrection plant Craterostigma plantagineum. A contribution to the study of drought tolerance at molecular level. Plant Physiology 127: 1346-1353.

Bartels D, Schneider K, Terstappen G, Piatkowski D, Salamini F. 1990. Molecular cloning of abscisic acid modulated genes which are induced during desiccation of the resurrection plant Craterostigma plantagineum. Planta 181: 27-34.

Beguiristain T, Grandbastien MA, Puigdomènech P, Casacuberta JC. 2001. Three Tnt1 subfamilies show different stress-associated patterns 
of expression in tobacco. Consequences for retrotransposon control and evolution in plants. Plant Physiology 127: 212-221.

Bernacchia G, Schwall G, Lottspeich F, Salamini F, Bartels D. 1995. The transketolase gene family of the resurrection plant Craterostigma plantagineum: differential expression during the rehydration phase. EMBO Journal 14: 610-618.

Brosius J. 2003. The contribution of RNAs and retroposition to evolutionary novelties. Genetica 118: 99-116.

Burkhardt RW. 1995. The spirit of system: Lamarck and evolutionary biology. Cambridge, MA, USA: Harvard University Press.

Dunoyer P, Himber C, Voinnet O. 2005. DICER-LIKE 4 is required for RNA interference and produces the 21-nucleotide small interfering RNA component of the plant cell-to-cell silencing signal. Nature Genetics 37: $1356-1360$.

Feschotte C, Jing N, Wessler SR. 2002. Plant transposable elements: where genetics meets genomics. Nature Reviews Genetics 3: 329-341.

Furini A, Koncz C, Salamini F, Bartels D. 1994. Agrobacterium-mediated transformation of the desiccation tolerant plant Craterostigma plantagineum. Plant Cell Reports 14: 102-106.

Furini A, Koncz C, Salamini F, Bartels D. 1997. High level transcription of a member of a repeated gene family confers dehydration tolerance to callus tissue of Craterostigma plantagineum. EMBO Journal 16: 3599-3608.

Hamilton A, Voinnet O, Chappell L, Baulcombe DC. 2002. Two classes of short interfering RNA in RNA silencing. EMBO Journal 21: 4671-4679.

Hamilton AJ, Baulcombe DC. 1999. A species of small antisense RNA in posttranscriptional gene silencing in plants. Science 286: 950-952.

Himber C, Dunoyer P, Moissiard G, Ritzenthaler C, Voinnet O. 2003. Transitivity-dependent and -independent cell-to-cell movement of RNA silencing. EMBO Journal 22: 4523-4533.

Hirotsune S, Yoshida N, Chen A, Garrett L, Sugiyama F, Takahashi S, Yagami K, Wynshaw-Boris A, Yoshiki A. 2003. An expressed pseudogene regulates the messenger-RNA stability of its homologous coding gene. Nature 423: 91-100.

Kalendar R, Tanskanen J, Immonen S, Nevo E, Schulman A. 2000. Genome evolution of wild barley (Hordeum spontaneum) by BARE-1 retrotransposon dynamics in response to sharp microclimatic divergence. Proceedings of the National Academy of Sciences, USA 97: 6603-6607.

Kao KN, Michayluk MR. 1975. Nutritional requirements for growth of Vicia hajastana cells and protoplasts at very low population density in liquid media. Planta 126: 105-110.

Kashkush K, Feldman M, Levy A. 2003. Transcriptional activation of retrotransposons alters the expression of adjacent genes in wheat. Nature Genetics 33: 102-106.

Koerber H, Strizhov N, Staiger D, Feldwisch J, Olsson O, Sandberg G, Palme K, Schell J, Koncz C. 1991. T-DNA gene 5 of Agrobacterium modulates auxin response by autoregulated synthesis of a growth hormone antagonist in plants. EMBO Journal 10: 3983-3991.

Koncz C, Martini N, Szabados L, Hrouda M, Bachmair A, Schell J. 1994. Specialized vectors for gene tagging and expression studies. In: Gelvin SB, Schilperoort RA, Verma DPS, eds. Plant molecular biology manual. Dordrecht, the Netherlands: Kluwer Academic Publishers, B2, 1-22.

Koncz C, Schell J. 1986. The promoter of the $\mathrm{T}_{\mathrm{L}}$-DNA gene 5 controls the tissue-specific expression of chimeric genes carried by a novel type of Agrobacterium binary vector. Molecular and General Genetics 204: 383-396.

Korneev SA, Park JH, O’Shea M. 1999. Neuronal expression of neural nitric oxide synthase (nNOS) protein is suppressed by an antisense RNA transcribed from an NOS pseudogene. Journal of Neuroscience 19: 7711-7722.

Krichevsky AM, Kosik KS. 2002. RNAi functions in cultured mammalian neurons. Proceedings of the National Academy of Sciences, USA 99: 11926-11929.
Kumar A, Bennetzen JL. 1999. Plant retrotransposons. Annual Review of Genetics 33: 479-532.

Lamarck JB. 1801, 1802. Discours donné dans le Muséum National d'Histoire Naturelle, l'an VIII de la République. Bulletin Scientifique de la France et de la Belgique 40: 443-595.

Liu B, Wendel JF. 2000. Retrotransposon activation followed by rapid repression in introgressed rice plants. Genome 43: 874-880.

Livak KJ, Schmittgen TD. 2001. Analysis of relative gene expression data using real-time quantitative PCR and the 2(-Delta Delta C(T)) method. Methods 25: 402-408.

Mallory AC, Vaucheret H. 2006. Functions of microRNAs and related small RNAs in plants. Nature Genetics Supplement 1 38: S31-36.

Mette MF, Aufsatz W, van der Winden J, Matzke MA, Matzke AJ. 2000. Transcriptional silencing and promoter methylation triggered by doublestranded RNA. EMBO Journal 19: 5194-5201.

Mhiri C, Morel JB, Vernhettes S, Casacuberta JM, Lucas H, Grandbastien MA. 1997. The promoter of the tobacco Tnt1 retrotransposon is induced by wounding and by abiotic stresses. Plant Molecular Biology 33: 257-266.

Nigumann P, Redik K, Mathik K, Speek M. 2002. Many human genes are transcribed from the antisense promoter of L1 retrotransposon. Genomics 79: 628-634.

Qi Y, Zhong X, Itaya A, Ding B. 2004. Dissecting RNA silencing in protoplasts uncovers novel effects of viral suppressors on the silencing pathway at the cellular level. Nucleic Acids Research 32: e179.

Raizada MN, Benito MI, Walbot V. 2001. The $M u D R$ transposon terminal inverted repeat contains a complex plant promoter directing distincting somatic and germinal programs. Plant Journal 25: 79-91.

Smith-Espinoza CJ, Phillips JR, Salamini F, Bartels D. 2005. Identification of further Craterostigma plantagineum cdt mutants affected in abscisic acid mediated desiccation tolerance. Molecular and General Genetics 274: 364-372.

Takeda S, Sugimoto K, Otsuki H, Hirochika H. 1999. A 13-bp cis-regulatory element in the LTR promoter of the tobacco retrotransposon Tto 1 is involved in responsiveness to tissue culture, wounding, methyl jasmonate and fungal elicitors. Plant Journal 18:383-393.

Vanitharani R, Chellappan P, Fauquet CM. 2003. Short interfering RNA-mediated interference of gene expression and viral DNA accumulation in cultured plant cells Proceedings of the National Academy of Sciences, USA 100: 9632-9636.

Varotto S, Locatelli S, Canova S, Pipal A, Motto M, Rossi V. 2003. Expression profile and cellular localization of maize Rpd3-type histone deacetylases during plant development. Plant Physiology 133: 606-617.

Vicient CM, Jaaskelainen MJ, Kalendar R, Schulman AH. 2001. Active retrotrasposons are a common feature of grass genomes. Plant Physiology 125: 1283-1292.

Voinnet O. 2004. Shaping small RNAs in plants by gene duplication. Nature Genetics 36: 1245-1246.

Voinnet O, Baulcombe DC. 1997. Systemic signalling in gene silencing. Nature 389: 553.

Voinnet O, Vain P, Angell S, Baulcombe DC. 1998. Systemic spread of sequence-specific transgene RNA degradation in plants is initiated by localized introduction of ectopic promoterless DNA. Cell 95: 177-187.

Wen-jun S, Forde BJ. 1989. Efficient transformation of Agrobacterium spp. by high voltage electroporation. Nucleic Acids Research 17: 8385.

Whitelaw E, Martin D. 2001. Retrotransposons as epigenetic mediators of phenotypic variation in mammals. Nature Genetics 27: 361-365.

Yamada K, Lim J, Dale JM, Chen H, Shinn P, Palm CJ, Southwick AM, Wu HC, Kim C, Nguyen M et al. 2003. Empirical analysis of transcriptional activity in the Arabidopsis genome. Science 302: 842-846.

Yoshikawa M, Peragine A, Park MY, Poethig RS. 2005. A pathway for the biogenesis of trans-acting siRNAs in Arabidopsis. Genes and Development 19: 2164-2175. 
New

Phytologist

\section{Supplementary Material}

The following supplementary material is available for this article online:

Fig. S1 Craterostigma plantagineum plant and calli.

Fig. S2 In situ hybridization with sense and antisense CDet-27-45 and sense CDT-1 riboprobes.

Fig. S3 Nucleotide sequence of the CDT-1 element.

Fig. S4 Phenotypes of calli transformed with constructs $a, b$, $\mathrm{c}$ and $\mathrm{d}$.
This material is available as part of the online article from: http://www.blackwell-synergy.com/doi/abs/10.1111/ j.1469-8137.2008.02480.x

(This link will take you to the article abstract.)

Please note: Blackwell Publishing are not responsible for the content or functionality of any supplementary materials supplied by the authors. Any queries (other than about missing material) should be directed to the journal at New Phytologist Central Office.

\section{About New Phytologist}

1

- New Phytologist is owned by a non-profit-making charitable trust dedicated to the promotion of plant science, facilitating projects from symposia to open access for our Tansley reviews. Complete information is available at www.newphytologist.org.

- Regular papers, Letters, Research reviews, Rapid reports and both Modelling/Theory and Methods papers are encouraged. We are committed to rapid processing, from online submission through to publication 'as-ready' via OnlineEarly - our average submission to decision time is just 29 days. Online-only colour is free, and essential print colour costs will be met if necessary. We also provide 25 offprints as well as a PDF for each article.

- For online summaries and ToC alerts, go to the website and click on 'Journal online'. You can take out a personal subscription to the journal for a fraction of the institutional price. Rates start at $£ 135$ in Europe/\$251 in the USA \& Canada for the online edition (click on 'Subscribe' at the website).

- If you have any questions, do get in touch with Central Office (newphytol@lancaster.ac.uk; tel +44 1524 594691) or, for a local contact in North America, the US Office (newphytol@ornl.gov; tel +1 865576 5261). 